\title{
Article
}

\section{Studies on the Mechanism of Catalysis of Iron\#Sulfur Cluster Transfer from IscU[2Fe2S] by HscA/HscB Chaperones}

Francesco Bonomi, Stefania lametti, Anna Morleo, Dennis Ta, and Larry E. Vickery Biochemistry, 2008, 47 (48), 12795-12801 • DOI: 10.1021/bi801565j • Publication Date (Web): 06 November 2008

Downloaded from http://pubs.acs.org on January 8, 2009
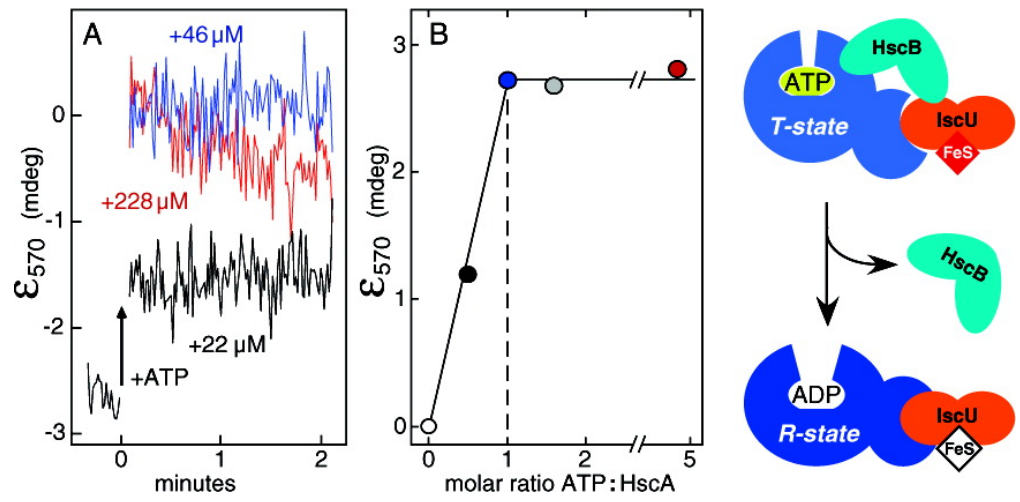

\section{More About This Article}

Additional resources and features associated with this article are available within the HTML version:

- $\quad$ Supporting Information

- Access to high resolution figures

- $\quad$ Links to articles and content related to this article

- $\quad$ Copyright permission to reproduce figures and/or text from this article

\section{View the Full Text HTML}




\title{
Studies on the Mechanism of Catalysis of Iron-Sulfur Cluster Transfer from IscU[2Fe2S] by $\mathrm{HscA} / \mathrm{HscB}$ Chaperones ${ }^{\dagger}$
}

\author{
Francesco Bonomi, ${ }^{*}$ Stefania Iametti, ${ }^{\ddagger}$ Anna Morleo, ${ }^{\star}$ Dennis Ta, ${ }^{\S}$ and Larry E. Vickery ${ }^{*}, 8$ \\ Section of Biochemistry, DISMA, University of Milan, Celoria 2, 20133 Milan, Italy, and Department of Physiology and \\ Biophysics, University of California, Irvine, California 92617
}

Received August 19, 2008; Revised Manuscript Received September 19, 2008

\begin{abstract}
The HscA/HscB chaperone/cochaperone system accelerates transfer of iron-sulfur clusters from the FeS-scaffold protein IscU (Isc $\mathrm{I}_{2}[2 \mathrm{Fe} 2 \mathrm{~S}]$, holo-IscU) to acceptor proteins in an ATP-dependent manner. We have employed visible region circular dichroism (CD) measurements to monitor chaperonecatalyzed cluster transfer from holo-IscU to apoferredoxin and to investigate chaperone-induced changes in properties of the $\mathrm{Isc}_{2}[2 \mathrm{Fe} 2 \mathrm{~S}]$ cluster. HscA-mediated acceleration of [2Fe2S] cluster transfer exhibited an absolute requirement for both $\mathrm{HscB}$ and ATP. A mutant form of HscA lacking ATPase activity, $\mathrm{HscA}(\mathrm{T} 212 \mathrm{~V})$, was unable to accelerate cluster transfer, suggesting that ATP hydrolysis and conformational changes accompanying the ATP (T-state) to ADP (R-state) transition in the HscA chaperone are required for catalysis. Addition of $\mathrm{HscA}$ and $\mathrm{HscB}$ to $\mathrm{Isc}_{2}[2 \mathrm{Fe} 2 \mathrm{~S}]$ did not affect the properties of the [2Fe2S] cluster, but subsequent addition of ATP was found to cause a transient change of the visible region CD spectrum, indicating distortion of the IscU-bound cluster. The dependence of the rate of decay of the observed CD change on ATP concentration and the lack of an effect of the HscA(T212V) mutant were consistent with conformational changes in the cluster coupled to ATP hydrolysis by HscA. Experiments carried out under conditions with limiting concentrations of HscA, HscB, and ATP further showed that formation of a 1:1:1 $\mathrm{HscA}-\mathrm{HscB}-\mathrm{Isc}_{2}[2 \mathrm{Fe} 2 \mathrm{~S}]$ complex and a single ATP hydrolysis step are sufficient to elicit the full effect of the chaperones on the $[2 \mathrm{Fe} 2 \mathrm{~S}]$ cluster. These results suggest that acceleration of iron-sulfur cluster transfer involves a structural change in the $\mathrm{Isc}_{2}[2 \mathrm{Fe} 2 \mathrm{~S}]$ complex during the $\mathrm{T} \Longrightarrow \mathrm{R}$ transition of HscA accompanying ATP hydrolysis.
\end{abstract}

The biosynthesis of iron-sulfur proteins is a multistep process involving a number of specialized proteins that mediate iron-sulfur cluster formation and delivery to acceptor proteins (reviewed in refs $1-5$ ). The most widely distributed and conserved system involves a scaffold protein employed for preassembly of FeS complexes and a Hsp70 class chaperone and J-protein cochaperone system that interacts with the scaffold protein (reviewed in ref 6 ). Specialized forms of Hsp70 and their cochaperones have evolved in bacteria ( $\mathrm{HscA}$ and $\mathrm{HscB}$ ) and in certain fungi (Ssq1 and Jac1), whereas most eukaryotes employ a multifunctional mitochondrial Hsp70 (mtHsp70) together with a specialized cochaperone homologous to $\mathrm{HscB} / \mathrm{Jac} 1$. HscA and Ssq1 have been shown to specifically bind to a conserved sequence present in the FeS-scaffold protein designated IscU in bacteria $(7,8)$ and Isu in fungi $(9,10)$, and the crystal structure of a complex of an IscU-derived peptide with the substrate binding domain of HscA has been determined (11). The interaction of IscU/Isu with HscA/Ssq1 is regulated by the cochaperone $\mathrm{HscB} / \mathrm{Jac} 1$ that binds the scaffold protein

\footnotetext{
† This work was supported in part by funds from the University of Milan (FIRST, to F.B.), by NIH Grant GM54264 (to L.E.V.), and by a postdoctoral fellowship from the University of Milan (to A.M.).

* To whom correspondence should be addressed. F.B.: e-mail, francesco.bonomi@unimi.it; tel, 39-02-50316819. L.E.V.: e-mail, LVickery@uci.edu; tel, 949-824-6580; fax, 949-824-8540.

₹University of Milan.

${ }^{\S}$ University of California, Irvine.
}

to assist its delivery to the chaperone. The cochaperone further stabilizes the chaperone-scaffold complex by enhancing chaperone ATPase activity to convert the lowaffinity chaperone-ATP complex to the higher affinity chaperone-ADP complex (12).

The exact functions chaperone-scaffold interactions play in cluster assembly/transfer events remain to be established. In vivo and in vitro studies of yeast Ssq1 and Jac1 indicated that the chaperones are not required for FeS cluster assembly on Isu, suggesting that the interactions instead play a role in cluster maturation and/or transfer to acceptor proteins $(13,14)$. In vitro studies using purified forms of bacterial $\mathrm{HscA}, \mathrm{HscB}$, and $\mathrm{IscU}$ have provided evidence that the chaperones act to facilitate cluster transfer. Bonomi et al. (15) found that Escherichia coli $\mathrm{HscA} / \mathrm{HscB}$ enhanced the rate of cluster transfer from $\mathrm{Isc}_{2}[2 \mathrm{Fe} 2 \mathrm{~S}]$ to apoferredoxin in an ATPdependent manner; moreover, stimulation occurred at low chaperone/scaffold ratios, suggesting that the chaperones act catalytically. Chandramouli and Johnson (16) carried out studies with the $\mathrm{HscA} / \mathrm{HscB} / \mathrm{IscU}$ system from Azotobacter vinelandii and found that stoichiometric concentrations of the chaperones together with excess ATP stimulated cluster transfer to apoferredoxin. Analysis of the $\mathrm{HscA} / \mathrm{HscB} / \mathrm{IscU}$ chaperone cycle (17) led Chandramouli and Johnson (16) to suggest that enhancement could result from interaction of acceptor proteins with the $\mathrm{HscA}(\mathrm{ATP})-\mathrm{HscB}-\mathrm{Isc}_{2}[2 \mathrm{Fe} 2 \mathrm{~S}]$ complex or alternatively with the $\mathrm{HscA}(\mathrm{ADP})-\mathrm{IscU}(2 \mathrm{Fe} 2 \mathrm{~S})$ 
complex formed following ATP hydrolysis. Because the conformational states of HscA with ATP bound (T-state) or ADP bound (R-state) differ, cluster release could be facilitated by structural alterations of $\mathrm{Isc}_{2}[2 \mathrm{Fe} 2 \mathrm{~S}]$ imposed by interactions with the HscA(ATP) T-state complex or by alterations coupled to conformational changes in $\mathrm{HscA}$ occurring during the $\mathrm{T} \Rightarrow \mathrm{R}$ transition following ATP hydrolysis. The rapid rate of ATP hydrolysis by the $\mathrm{HscA}(\mathrm{ATP})-\mathrm{HscB}-\mathrm{Isc}_{2}[2 \mathrm{Fe} 2 \mathrm{~S}]$ complex makes it difficult to distinguish between these two possible mechanisms.

In the studies described herein we have used a mutant form of HscA lacking ATPase activity to investigate the role of ATP hydrolysis in facilitating cluster transfer. The findings suggest that structural changes in the $\mathrm{Isc}_{2}[2 \mathrm{Fe} 2 \mathrm{~S}]$ complex that occur during the $\mathrm{T} \Rightarrow \mathrm{R}$ conformational change of HscA accompanying ATP hydrolysis are coupled to [2Fe2S] cluster activation and subsequent transfer.

\section{EXPERIMENTAL PROCEDURES}

Proteins. Recombinant forms of E. coli IscU (12), ferredoxin (18), HscA (20), and HscB (20) were prepared according to previously published procedures, and protein concentrations were determined as described therein. HscA(T212V) was prepared by site-specific mutagenesis (QuikChange; Stratagene) using the pTrcHsc66 plasmid (20), and mutations were confirmed by DNA sequencing (Laragen Inc.). The HscA(T212V) protein was expressed and purified as for wild-type HscA (20). Concentrations of the holo forms of IscU and ferredoxin are reported in terms of the [2Fe2S] complex of each protein. Under the conditions employed $\mathrm{Isc} \mathrm{U}$ behaves as a dimer, and concentrations are therefore reported in terms of the $(\operatorname{IscU})_{2}[2 \mathrm{Fe} 2 \mathrm{~S}]$ complex $(12,21)$; ferredoxin is monomeric and contains a single [2Fe2S] cluster (18).

Sample Handling and Cluster Reconstitution. Sample manipulations, including reagent preparation and chromatography, were carried out anaerobically under argon in septum-capped vials, cuvettes, or columns, and stainless steel needles, tubing, and cannulae were used for sample transfers as described previously (21). The buffer used was $0.1 \mathrm{M}$ Tris-HCl, pH 8.0, containing $5 \mathrm{mM}$ dithiothreitol (TD buffer). ${ }^{1}$ Where indicated, in experiments involving HscA and $\mathrm{HscB}, 10 \mathrm{mM} \mathrm{MgCl} 2$ and $150 \mathrm{mM} \mathrm{KCl}$ were added to TD buffer (referred to as TDMK buffer). Unless otherwise specified, the temperature was maintained at $23{ }^{\circ} \mathrm{C}$.

$\mathrm{Isc}_{2}[2 \mathrm{Fe} 2 \mathrm{~S}]$ was prepared by three sequential additions at $5 \mathrm{~min}$ intervals of 0.32 equiv of ferric ammonium citrate and lithium sulfide to $0.5 \mathrm{mM}$ apo-IscU in TD buffer on ice. The final solution thus contained slightly substoichiometric concentrations of iron and sulfide required for formation of holo-IscU. Formation of the [2Fe2S] cluster was monitored by visible region $\mathrm{CD}$ spectroscopy. At concentrations in the $0.5 \mathrm{mM}$ range reconstituted $\mathrm{Isc}_{2}[2 \mathrm{Fe} 2 \mathrm{~S}]$ was found to be stable for at least $18 \mathrm{~h}$ under anaerobic conditions in TD buffer at $0{ }^{\circ} \mathrm{C}$.

Native ferredoxin was isolated as the $[2 \mathrm{Fe} 2 \mathrm{~S}]$ complex (18). Apoferredoxin was prepared by precipitating the

\footnotetext{
${ }^{1}$ Abbreviations: CD, circular dichroism; TD buffer, $0.1 \mathrm{M}$ Tris$\mathrm{HCl}, \mathrm{pH}$ 8.0, and $5 \mathrm{mM}$ dithiothreitol; TDMK buffer, $0.1 \mathrm{M}$ Tris- $\mathrm{HCl}$, $\mathrm{pH}$ 8.0, $5 \mathrm{mM}$ dithiothreitol, $10 \mathrm{mM} \mathrm{MgCl}$, and $150 \mathrm{mM} \mathrm{KCl}$.
}

holoprotein in $10 \%$ trichloroacetic acid containing $10 \mathrm{mM}$ DTT for $10 \mathrm{~min}$ at $0{ }^{\circ} \mathrm{C}$. The apoferredoxin pellet was collected by centrifugation, washed twice in cold water under anaerobic conditions, and dissolved anaerobically in TD buffer.

Cluster Transfer Studies. Unless otherwise specified, reactions were initiated by addition of $\operatorname{Isc}_{2}[2 \mathrm{Fe} 2 \mathrm{~S}]$ to a solution containing a $1.05-1.10$ equiv of apoferredoxin in TDMK buffer at $23{ }^{\circ} \mathrm{C}$. Where indicated, HscA and/or HscB were (was) added to the buffer prior to other proteins. ATP was added from a $100 \mathrm{mM}$ stock solution in TDMK buffer immediately after the addition of $\operatorname{Isc}_{2}[2 \mathrm{Fe} 2 \mathrm{~S}]$. Mixtures were prepared directly in septum-capped $1 \mathrm{~mL}$ anaerobic cuvettes. Initial cluster transfer rates were calculated from the increase in ellipticity at $435 \mathrm{~nm}$ during the first $30-60$ s.

Chaperone-Induced Spectral Modifications. Unless otherwise specified, experiments were performed in TDMK buffer at $23{ }^{\circ} \mathrm{C}$ in septum-capped $1 \mathrm{~mL}$ anaerobic cuvettes. Spectral changes resulting from addition of $\mathrm{HscA}, \mathrm{HscB}$, and/ or ATP to $\mathrm{Isc}_{2}[2 \mathrm{Fe} 2 \mathrm{~S}]$ were monitored by $\mathrm{CD}$ by recording spectra at specified time intervals or by continuous monitoring of ellipticity at $570 \mathrm{~nm}$. Typically, $\mathrm{Isc}_{2}[2 \mathrm{Fe} 2 \mathrm{~S}]$ was mixed with a variable amount of $\mathrm{HscA}$ and $\mathrm{HscB}$, and the reaction was initiated by the addition of ATP. In some experiments ATP was added to mixtures of $\mathrm{Isc}_{2}[2 \mathrm{Fe} 2 \mathrm{~S}]$ and $\mathrm{HscA}$, and the reaction was initiated shortly thereafter by the addition of HscB.

Analytical Methods. ATPase assays (12), analyses for iron and sulfide (21), isothermal titration calorimetry measurements (22), and difference spectroscopy (22) were carried out as described previously. Circular dichroism measurements were recorded at $23^{\circ} \mathrm{C}$ in $1 \mathrm{~cm}$ path length anaerobic cuvettes using a Jasco J-810 spectropolarimeter and analyzed using Jasco software.

\section{RESULTS}

Circular Dichroism Spectra of Holo-IscU and Holoferredoxin. In the experiments described below we used E. coli apoferredoxin as an acceptor protein for $\mathrm{FeS}$ clusters provided by $\mathrm{Isc}_{2}[2 \mathrm{Fe} 2 \mathrm{~S}]$ and employed $\mathrm{CD}$ spectroscopy to monitor cluster transfer. Figure 1 shows the visible region $\mathrm{CD}$ spectra of $\mathrm{Isc}_{2}[2 \mathrm{Fe} 2 \mathrm{~S}]$, native holoferredoxin, and holoferredoxin reconstituted by cluster transfer from $\mathrm{Isc}_{2}[2 \mathrm{Fe} 2 \mathrm{~S}]$ in the presence of $\mathrm{HscA}, \mathrm{HscB}$, and ATP. The spectra of native and reconstituted holoferredoxin are virtually identical, suggesting that the structure of the $[2 \mathrm{Fe} 2 \mathrm{~S}]$ cluster in the reconstituted protein is the same as that of the native protein. This finding is similar to the earlier results involving $\mathrm{FeS}$ cluster transfer using $\mathrm{Isc}_{2}[2 \mathrm{Fe} 2 \mathrm{~S}]$ in the absence of chaperones (21) and indicates that under these conditions $\mathrm{HscA}$ and $\mathrm{HscB}$ do not alter the type of cluster transferred. In subsequent experiments cluster transfer from $\mathrm{Isc}_{2}[2 \mathrm{Fe} 2 \mathrm{~S}]$ to apoferredoxin was followed by monitoring ellipticity changes at $435 \mathrm{~nm}$.

FeS Cluster Transfer Studies. The rates of cluster transfer from $\mathrm{Isc}_{2}[2 \mathrm{Fe} 2 \mathrm{~S}]$ to apoferredoxin were determined under conditions including or lacking each chaperone protein and/ or ATP (Figure 2). Acceleration of cluster transfer was observed only when $\mathrm{HscA}, \mathrm{HscB}$, and ATP were all present, and omission of any component gave rates comparable to 


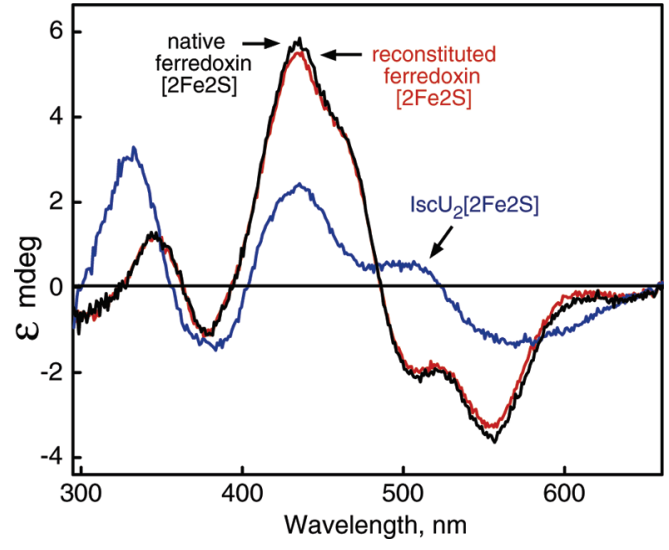

FIGURE 1: Circular dichroism spectra of holo-IscU and holoferredoxin. $\mathrm{Isc}_{2}[2 \mathrm{Fe} 2 \mathrm{~S}], 50 \mu \mathrm{M}$, was prepared by reconstitution of the apoprotein. Native ferredoxin[2Fe $2 \mathrm{~S}$ ], $48 \mu \mathrm{M}$, was purified directly from cultures of $E$. coli. Reconstituted ferredoxin[2Fe2S] was prepared by incubating $48 \mu \mathrm{M}$ apoferredoxin, $38 \mu \mathrm{M}$ HscA, 38 $\mu \mathrm{M} \mathrm{HscB}$, and $50 \mu \mathrm{M} \operatorname{Isc}_{2}[2 \mathrm{Fe} 2 \mathrm{~S}]$ with $2 \mathrm{mM}$ ATP for $4 \mathrm{~h}$ in TDMK buffer at $23{ }^{\circ} \mathrm{C}$.

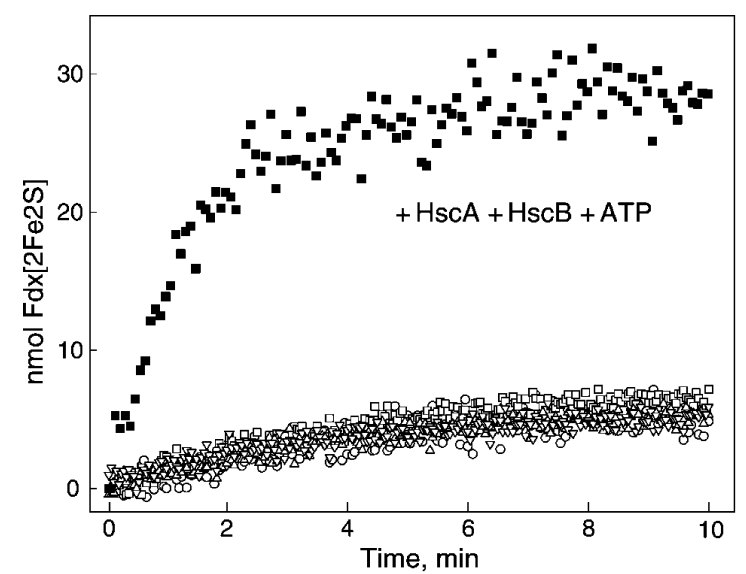

FIGURE 2: Rates of [2Fe2S] cluster transfer from $\mathrm{Isc}_{2}[2 \mathrm{Fe} 2 \mathrm{~S}]$ to apoferredoxin. Cluster transfer was assessed by monitoring CD ellipticity increases at $435 \mathrm{~nm}$ following the addition of $50 \mu \mathrm{M}$ $\mathrm{IscU}_{2}[2 \mathrm{Fe} 2 \mathrm{~S}]$ to $48 \mu \mathrm{M}$ apoferredoxin. Other additions: none $(\mathrm{O})$; $\mathrm{HscA}, \mathrm{HscB}$, and ATP $(\square)$; HscA and HscB $(\triangle)$; HscA and ATP $(\nabla)$; ATP $(\square)$. When present, HscA and $\mathrm{HscB}$ were $149 \mu \mathrm{M}$ each; ATP was added at a final concentration of $2 \mathrm{mM}$ immediately before the addition of $\mathrm{IscU}_{2}[2 \mathrm{Fe} 2 \mathrm{~S}]$.

the background rate of transfer observed using $\mathrm{Isc}_{2}[2 \mathrm{Fe} 2 \mathrm{~S}]$ alone. The finding that no inhibition was observed in the presence of $\mathrm{HscA}$ with or without $\mathrm{HscB}$ in the absence of ATP indicates that simple reversible binding of $\mathrm{Isc}_{2}[2 \mathrm{Fe} 2 \mathrm{~S}]$ to the chaperone(s) does not affect the rate of cluster transfer. Although these findings establish that ATP is required for chaperone enhancement of cluster transfer, they do not allow determination of whether ATP hydrolysis is required or whether ATP binding to the $\left.\mathrm{HscA}-\mathrm{HscB}-\mathrm{IscU}_{2} 2 \mathrm{Fe} 2 \mathrm{~S}\right]$ complex is sufficient.

The effect of chaperone concentration on the initial rate of FeS cluster transfer was initially studied using an excess of ATP. Figure 3A shows that the rate of cluster transfer increases linearly as the amount of $\mathrm{HscA}$ and equivalent $\mathrm{HscB}$ are increased over the range to where there is one $\mathrm{HscA}$ and $\mathrm{HscB}$ per IscU monomer equvalent. The rates observed correspond to $\cong 0.1 \mathrm{~mol}$ of holoferredoxin formed ( $\mathrm{mol}$ of $\mathrm{HscA})^{-1} \mathrm{~min}^{-1}$, an acceleration of approximately 10-fold over the background rate. At each concentration of chaperone tested CD spectra recorded at equilibrium $(4-18 \mathrm{~h})$ showed

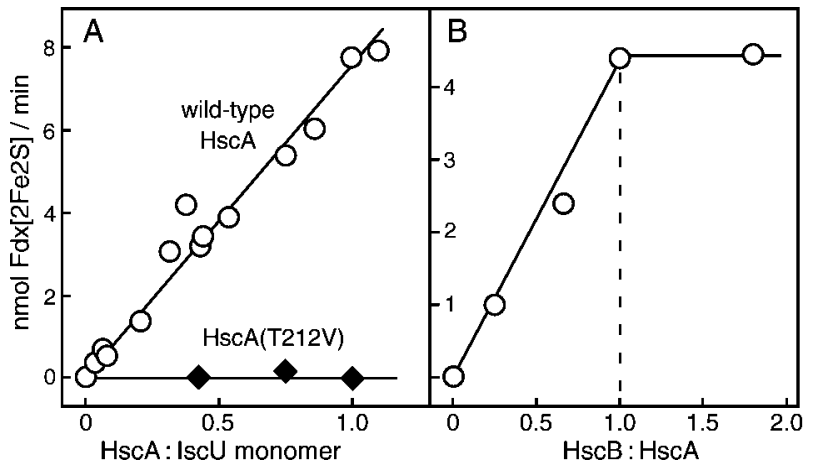

FiguRE 3: Dependence of initial cluster transfer rate from $\mathrm{Isc}_{2}[2 \mathrm{Fe} 2 \mathrm{~S}]$ to apoferredoxin on $\mathrm{HscA}$ and $\mathrm{HscB}$ ratios. Rates were calculated from the increase in CD ellipticity at $435 \mathrm{~nm}$, and values were corrected for background cluster transfer observed in the absence of $\mathrm{HscA}$ and $\mathrm{HscB}$. Conditions are similar to those given in Figure 2 except that the concentrations of $\mathrm{HscA}$ and $\mathrm{HscB}$ were varied. ATP was added at a final concentration of $2 \mathrm{mM}$ immediately before the addition of $\operatorname{Isc}_{2}[2 \mathrm{Fe} 2 \mathrm{~S}]$. Left panel: The concentration of $\mathrm{Isc}_{2}[2 \mathrm{Fe} 2 \mathrm{~S}]$ was held constant, and the concentration of $\mathrm{HscA}(\mathrm{O})$ or $\mathrm{HscA}(\mathrm{T} 212 \mathrm{~V})(\diamond)$ and equivalent concentrations of $\mathrm{HscB}$ were varied. Results are plotted in terms of the concentration of the IscU monomer. Right panel: The concentrations of $\mathrm{HscA}$ and $\mathrm{IscU}_{2}[2 \mathrm{Fe} 2 \mathrm{~S}]$ were held constant, and the concentrations of $\mathrm{HscB}$ was varied.

that cluster transfer was complete and quantitative, indicating that the chaperones affect the rate but not the yield of transfer.

Figure $3 \mathrm{~B}$ shows that the cochaperone $\mathrm{HscB}$ is essential for activity. Previous studies showed that HscB and apoIscU form a ternary complex with $\mathrm{HscA}$ (ATP) (17), and the finding that acceleration of $\mathrm{FeS}$ cluster transfer increases linearly with the amount of $\mathrm{HscB}$ until a concentration equivalent to $\mathrm{HscA}$ is reached is consistent with a requirement for formation of a $\mathrm{HscA}(\mathrm{ATP})-\mathrm{HscB}-\mathrm{Isc}_{2}$ [2Fe2S] complex for activity. However, it is not known whether the $\mathrm{HscB}$ requirement arises from a direct effect on the structure of the ternary T-state complex prior to ATP hydrolysis or reflects synergistic stimulation of HscA ATPase activity by the cochaperone.

Requirement for ATP Hydrolysis. To determine whether formation of the HscA(ATP) $-\mathrm{HscB}-\mathrm{Isc}_{2}[2 \mathrm{Fe} 2 \mathrm{~S}] \mathrm{T}$-state complex was sufficient to facilitate cluster transfer or whether ATP hydrolysis and the resulting $\mathrm{T} \Longrightarrow \mathrm{R}$ conformational transition are required, we employed a mutant of $\mathrm{HscA}$ lacking ATPase activity. Earlier studies with the E. coli Hsp70 chaperone DnaK $(22,23)$ showed that mutation of a conserved threonine residue in the nucleotide binding site reduced ATPase activity more than $90 \%$. Thr-212 occupies a similar position in the nucleotide binding site of $\mathrm{HscA},{ }^{2}$ and we replaced Thr-212 with valine using site-specific mutagenesis. The $\mathrm{HscA}(\mathrm{T} 212 \mathrm{~V})$ mutant did not exhibit measurable ATPase activity $(<0.01 \mathrm{~mol}$ of ATP hydrolyzed $\min ^{-1}$ ), and activity could not be detected in the presence of $\mathrm{HscB}$ or IscU or mixtures of $\mathrm{HscB}$ and IscU that stimulate the ATPase activity of wild-type HscA $>400$-fold. ATP was able to bind to $\operatorname{HscA}(\mathrm{T} 212 \mathrm{~V})$ as addition of ATP to the mutant elicited a near-UV difference spectrum indicative of T-state formation as observed with wild-type HscA (24) (see Supporting Information Figure S1). However, in contrast to wild-type HscA the ATP-induced difference spectrum re-

\footnotetext{
${ }^{2}$ J. R. Cupp-Vickery, P. C. Aoto, D. T. Ta, and L. E. Vickery, unpublished results.
} 


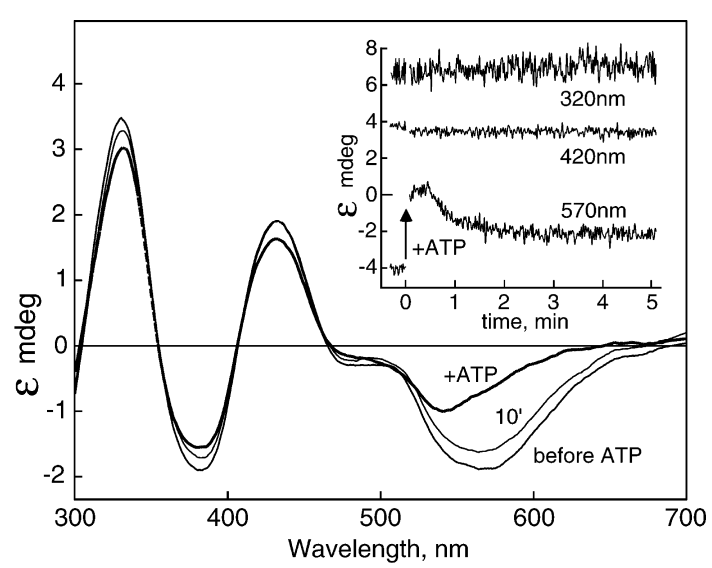

FIGURE 4: Transient effects of HscA, HscB, and ATP on the CD spectrum of $\mathrm{IscU}_{2}[2 \mathrm{Fe} 2 \mathrm{~S}]$. Samples contained $54 \mu \mathrm{M} \mathrm{IscU}_{2}[2 \mathrm{Fe} 2 \mathrm{~S}]$, $113 \mu \mathrm{M} \mathrm{HscA}$, and $125 \mu \mathrm{M} \mathrm{HscB}$ in TDMK buffer at $23{ }^{\circ} \mathrm{C}$. Spectra were recorded before and immediately or 10 min after the addition of $2 \mathrm{mM}$ ATP. The scan rate was $100 \mathrm{~nm} / \mathrm{min}$. Inset: Time course of ellipticity changes at selected wavelengths following addition of ATP.

mained stable for $>30$ min, consistent with loss of ATPase activity. Whereas ATP hydrolysis was defective, IscU binding to $\mathrm{HscA}(\mathrm{T} 212 \mathrm{~V})$ appeared normal. Isothermal titration calorimetry experiments yielded a $K_{\mathrm{d}}$ of $1.5 \mu \mathrm{M}$ for Isc $(n=1.0)$ in the presence of $1 \mathrm{mM}$ ADP compared to $1.6 \mu \mathrm{M}$ observed for wild-type HscA (25) (see Supporting Information Figure S2).

Studies on the ability of the $\mathrm{HscA}(\mathrm{T} 212 \mathrm{~V})$ mutant to accelerate FeS cluster transfer from IscU to apoferredoxin were carried out in a manner similar to the experiments with wild-type HscA. As shown in Figure 3A ( ) HscA(T212V) had no significant effect on the rate of holoferredoxin formation. Cluster transfer was neither enhanced nor inhibited at $\mathrm{HscA}(\mathrm{T} 212 \mathrm{~V})$ concentrations up to 1 equiv per IscU monomer. These results strongly suggest that formation of the $\mathrm{HscA}(\mathrm{ATP})-\mathrm{HscB}-\mathrm{Isc}_{2}[2 \mathrm{Fe} 2 \mathrm{~S}] \mathrm{T}$-state complex is not sufficient to facilitate cluster transfer and that ATP hydrolysis and subsequent conformational changes in $\mathrm{HscA}$ are required.

Chaperone Effects on Isc $U_{2}[2 \mathrm{Fe} 2 \mathrm{~S}]$. We used CD spectroscopy to investigate whether the interaction of $\mathrm{Isc}_{2}[2 \mathrm{Fe} 2 \mathrm{~S}]$ with $\mathrm{HscA}$ and $\mathrm{HscB}$ might have effects on the properties of the $[2 \mathrm{Fe} 2 \mathrm{~S}]$ cluster. Addition of $\mathrm{HscA}$ or $\mathrm{HscB}$ alone or each individually in combination with ATP had no detectable effect on the visible region $\mathrm{CD}$ of the [2Fe2S] cluster (data not shown). However, addition of HscA and $\mathrm{HscB}$ together with ATP caused a transient change in the $\mathrm{CD}$ spectrum in the region from 520 to $620 \mathrm{~nm}$. Figure 4 shows spectra recorded prior to, immediately after, and 10 min after addition of ATP. The spectral scan recorded immediately following ATP addition displays significant loss of the $\mathrm{CD}$ trough observed near $570 \mathrm{~nm}$, whereas the scan initiated 10 min after ATP addition shows only a small loss of signal. The time dependence of the change observed suggested that the effects on the cluster are transient, and full effects at shorter wavelengths may not have been observed due to the time required to complete the scan. For this reason, the kinetics of $\mathrm{CD}$ changes were followed at wavelengths of 320,420 , and $570 \mathrm{~nm}$ corresponding to extrema in the CD spectrum. As shown in the inset to Figure 4 , the largest change was associated with the trough near $570 \mathrm{~nm}$. The CD signal was reduced to near zero shortly

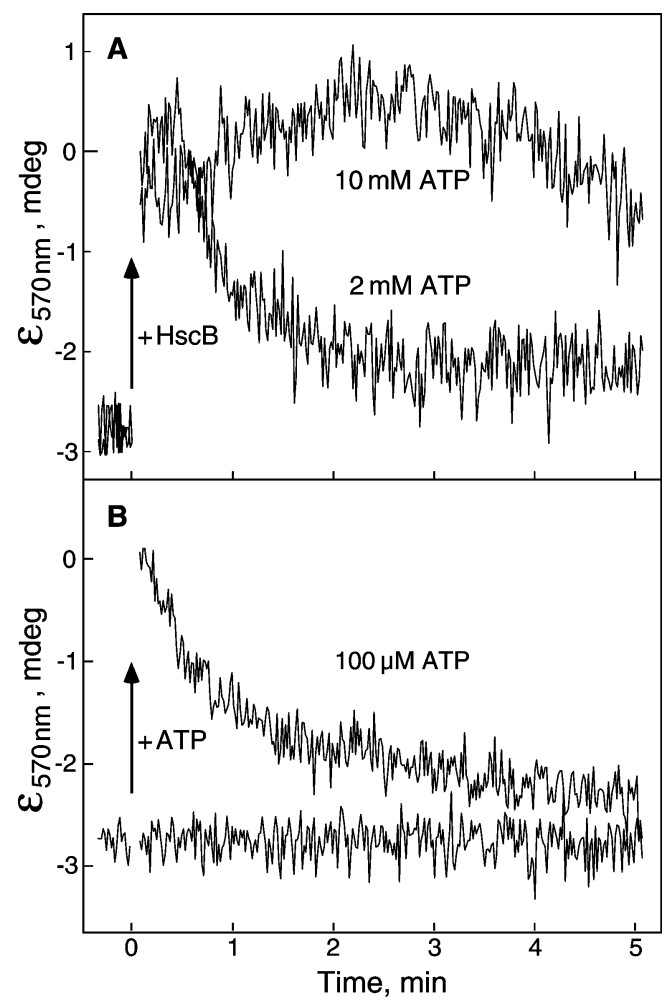

FIGURE 5: Effect of ATP concentration on induced CD changes of $(\mathrm{IscU})_{2}[2 \mathrm{Fe} 2 \mathrm{~S}]$. Panel A: Sample mixtures contained $54 \mu \mathrm{M}$ $\mathrm{IscU}_{2}[2 \mathrm{Fe} 2 \mathrm{~S}]$ and $113 \mu \mathrm{M} \mathrm{HscA}$, and 2 or $10 \mathrm{mM}$ ATP in TDMK buffer at $20{ }^{\circ} \mathrm{C}$. Reactions were initiated by addition of $125 \mu \mathrm{M}$ $\mathrm{HscB}$, and ellipticity changes were monitored at $570 \mathrm{~nm}$. Panel B: The sample in the upper trace contained $54 \mu \mathrm{M} \mathrm{IscU}_{2}[2 \mathrm{Fe} 2 \mathrm{~S}]$ and $122 \mu \mathrm{M} \mathrm{HscA}$ and HscB in TDMK buffer at $23^{\circ} \mathrm{C}$, and the reaction was initiated by addition of $100 \mu \mathrm{M}$ ATP. The sample in the lower trace lacked HscA and HscB.
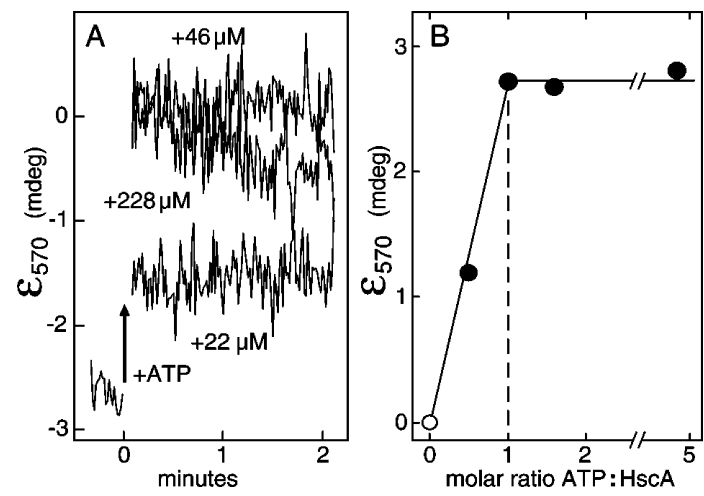

FIGURE 6: Effect of stoichiometric concentrations of HscA, HscB, and ATP on induced $\mathrm{CD}$ changes of $\mathrm{IscU}_{2}[2 \mathrm{Fe} 2 \mathrm{~S}]$. Samples contained $54 \mu \mathrm{M} \mathrm{Isc}_{2}[2 \mathrm{Fe} 2 \mathrm{~S}]$ and $48 \mu \mathrm{M}$ HscA and $\mathrm{HscB}$ in TDMK buffer at $20{ }^{\circ} \mathrm{C}$. Panel A: Time course of ATP-induced changes. ATP was added to give final concentrations of 22, 46, or $222 \mu \mathrm{M}$ corresponding to $0.46,0.96$, or 4.6 equiv of $\mathrm{HscA}$, respectively. Panel B: Effects of the ratio of ATP to HscA. The maximum amplitude of $\Delta \varepsilon_{570}$ observed in panel $\mathrm{A}$ is plotted as a function of the molar ratio of ATP to HscA. The shaded circle at a molar ratio of 1.6 corresponds to the ellipticity change observed using $62 \mu \mathrm{M}$ HscA and HscB plus $100 \mu \mathrm{M}$ ATP in Figure 5B.

following addition of ATP and reappeared slowly over the next several minutes.

To test whether the $\mathrm{CD}$ change observed required ATP hydrolysis, we carried out similar CD experiments with the HscA(T212V) mutant. No time-dependent spectral changes were observed using $\mathrm{HscA}(\mathrm{T} 212 \mathrm{~V})$, indicating that hydroly- 


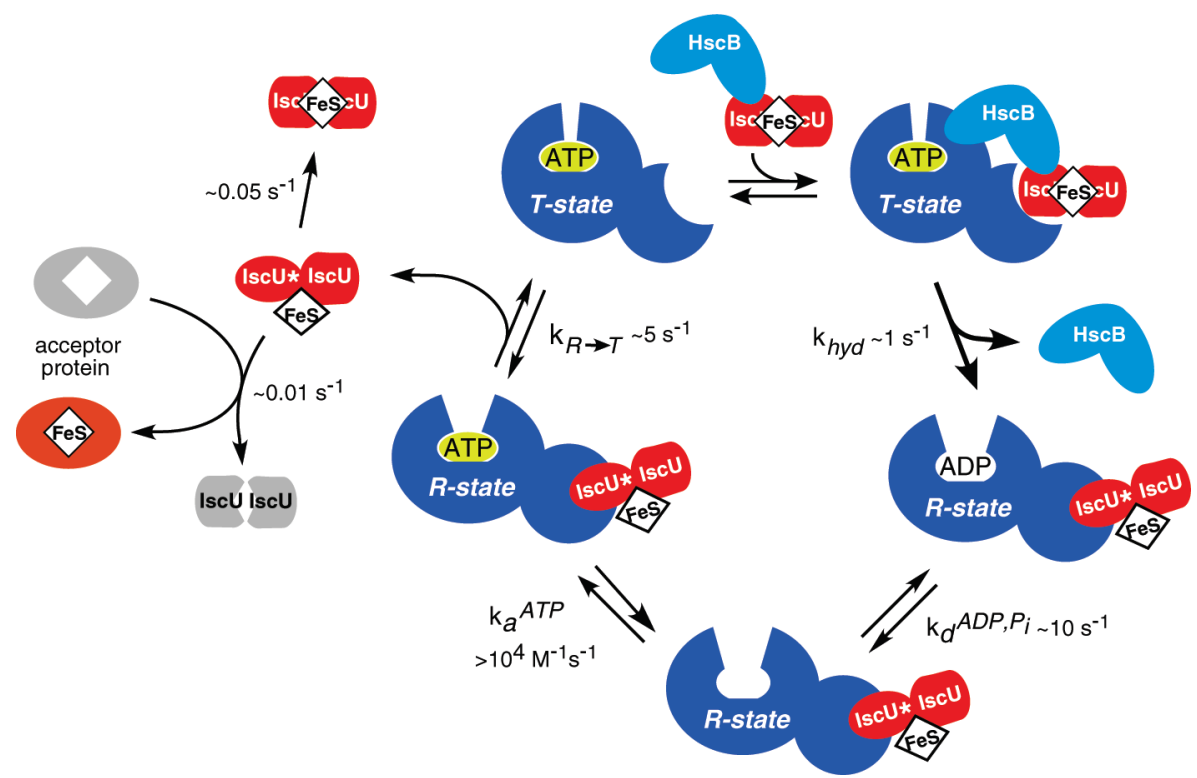

FIGURE 7: Proposed mechanistic scheme for chaperone-catalyzed FeS cluster transfer from $\mathrm{Isc}_{2}[2 \mathrm{Fe} 2 \mathrm{~S}]$ to apoacceptor proteins. The HscA ATPase reaction cycle scheme and rates shown are derived from ref 17. See text for discussion.

sis of ATP is necessary and the formation of an $\mathrm{HscA}(\mathrm{ATP})-\mathrm{HscB}-\mathrm{IscU}_{2}[2 \mathrm{Fe} 2 \mathrm{~S}]$ complex is not sufficient to elicit the effect (data not shown). The finding that ATP hydrolysis is required suggested that the time-dependent recovery of the $\mathrm{CD}$ signal may result from ATP depletion in the system. HscB and IscU synergistically stimulate HscA ATPase activity $>400$ fold to give rates of $\sim 1 \mathrm{~s}^{-1}$, and under the high chaperone concentrations employed $2 \mathrm{mM}$ ATP would be rapidly hydrolyzed. To test this possibility, we compared the rate of recovery of the $570 \mathrm{~nm} C D$ signal in the presence of 2 and $10 \mathrm{mM} \mathrm{ATP}$; in this experiment reactions were initiated by addition of $\mathrm{HscB}$. As shown in Figure 5A at the higher ATP level the effect on the CD signal is longer lived, consistent with recovery of the signal corresponding to ATP depletion. To test whether lower concentrations of ATP were sufficient to affect the change, we carried out the measurements under limited turnover conditions. The ATP concentration was reduced to $100 \mu \mathrm{M}$, greater than the concentration of $\mathrm{IscU}_{2}[2 \mathrm{Fe} 2 \mathrm{~S}](54 \mu \mathrm{M})$ but slightly less than the concentration of HscA $(122 \mu \mathrm{M})$. As shown in Figure 5B the $570 \mathrm{~nm}$ CD signal was completely lost even under these conditions of limited ATP.

The finding that the full effect of the chaperones on the $\mathrm{IscU}_{2}[2 \mathrm{Fe} 2 \mathrm{~S}] \mathrm{CD}$ signal could be obtained using quasi-single turnover conditions suggested that a single ATPase cycle involving one $\mathrm{HscA}(\mathrm{ATP})-\mathrm{HscB}-\mathrm{IscU}_{2}[2 \mathrm{Fe} 2 \mathrm{~S}]$ complex might elicit the effect. We tested this possibility by incubating IscU $_{2}[2 \mathrm{Fe} 2 \mathrm{~S}](54 \mu \mathrm{M})$ with a slightly substoichiometric amount of HscA and HscB (48 $\mu \mathrm{M}, 0.9$ equiv) and 4.6, 0.96, or 0.46 equiv of ATP per HscA. Figure 6 shows that a single equivalent of $\mathrm{HscA}, \mathrm{HscB}$, and ATP is sufficient to fully affect the ellipticity change observed at $570 \mathrm{~nm}$. Decreasing the amount of ATP below 1 equiv to 0.46 reduces the effect correspondingly. These results indicate that a single ATPase cycle involving $1 \mathrm{~mol}$ of $\mathrm{HscA}$ and $\mathrm{HscB} / \mathrm{mol}$ of $\mathrm{IscU}_{2}[2 \mathrm{Fe} 2 \mathrm{~S}]$ is sufficient to cause the changes observed in the CD spectrum of the FeS cluster.

\section{DISCUSSION}

The results described herein corroborate earlier findings of Bonomi et al. (15) and Chandramouli and Johnson (16) regarding ATP-dependent chaperone acceleration of [2Fe2S] cluster transfer from $\mathrm{Isc}_{2}[2 \mathrm{Fe} 2 \mathrm{~S}]$ to apoferredoxin. The maximal rate observed, $\cong 0.1 \mathrm{~mol}$ of holoferredoxin formed (mol of HscA $)^{-1} \mathrm{~min}^{-1}$, corresponds to a roughly 10 -fold enhancement of rates observed in the absence of HscA, HscB, and/or ATP. Furthermore, acceleration observed using less than stoichiometric amounts of $\mathrm{HscA}$ and $\mathrm{HscB}$ yielded complete formation of holoferredoxin, consistent with a catalytic role for the chaperones in cluster transfer. The requirement for $\mathrm{HscB}$ and $\mathrm{ATP}$ suggests that a ternary $\mathrm{HscA}(\mathrm{ATP})-\mathrm{HscB}-\mathrm{IscU}_{2}[2 \mathrm{Fe} 2 \mathrm{~S}]$ complex plays a critical role in activation of $\mathrm{Isc}_{2}[2 \mathrm{Fe} 2 \mathrm{~S}]$ for cluster transfer. As pointed out by Chandramouli and Johnson (16) activation could occur as a result of formation of the ternary complex itself or could occur during subsequent conformational changes coupled to ATP hydrolysis (cf. Figure 7). By employing a mutant form of HscA lacking ATPase activity, we found that ATP hydrolysis is necessary for the acceleration, suggesting that structural changes during the $\mathrm{T} \Longrightarrow \mathrm{R}$ conformational transition of $\mathrm{HscA}$ are coupled to the activation process.

The mechanism whereby the chaperones enhance [2Fe2S] cluster transfer is not known. Previous cluster transfer studies conducted in the absence of chaperone indicated that the cluster remains intact during transfer and that transfer likely involves direct protein interactions between $\mathrm{IscU}_{2}[2 \mathrm{Fe} 2 \mathrm{~S}]$ and the apoprotein acceptor. Studies by Cowan and coworkers (26) using human Isu and apoferredoxin showed that free iron in solution does not exchange with the cluster during transfer, indicating that there is not a significant degree of cluster disassembly and reassembly, and kinetic studies by Bonomi et al. (21) on cluster transfer from $\mathrm{IscU}_{2}[2 \mathrm{Fe} 2 \mathrm{~S}]$ to apoferredoxin provided evidence for formation of a donor-acceptor complex during transfer. Analysis of the kinetics of cluster formation and transfer further suggested that a labile FeS-scaffold species formed during initial cluster 
assembly on IscU was more efficiently transferred than that of the mature $\mathrm{Isc}_{2}[2 \mathrm{Fe} 2 \mathrm{~S}]$ complex (21). A similar labile species produced from the mature cluster during the chaperone ATPase cycle might also facilitate transfer, and the transient change observed in the $\mathrm{CD}$ spectrum of $\mathrm{Isc}_{2}[2 \mathrm{Fe} 2 \mathrm{~S}]$ upon incubation with $\mathrm{HscA}, \mathrm{HscB}$, and $\mathrm{ATP}$ may reflect formation of such an activated complex. The requirements for both $\mathrm{HscB}$ and ATP and the lack of activity of the $\mathrm{HscA}(\mathrm{T} 212 \mathrm{~V})$ mutant in elliciting the $\mathrm{CD}$ change are consistent with the observation that acceleration of cluster transfer occurs during the $\mathrm{R} \Rightarrow \mathrm{T}$ transition of $\mathrm{HscA}$ coincident with ATP hydrolysis. In the scheme presented in Figure 7 we have designated the form of IscU that gives rise to the altered $[2 \mathrm{Fe} 2 \mathrm{~S}]$ cluster $\mathrm{CD}$ spectrum as $\mathrm{IscU}^{*}$. This altered complex, or a species derived from it, may be the form of the scaffold cluster that exhibits an enhanced rate of transfer.

The molecular basis of the effect of $\mathrm{HscA}$ and $\mathrm{HscB}$ on $\mathrm{Isc}_{2}[2 \mathrm{Fe} 2 \mathrm{~S}]$ is not understood. Because the chaperones interact with the IscU protein, the effects on the $[2 \mathrm{Fe} 2 \mathrm{~S}]$ cluster are assumed to be indirect, i.e., mediated by structural changes induced in the scaffold protein rather than by direct interaction of the chaperones with the cluster. The chaperones could act by causing changes in IscU conformation that facilitate cluster release or capture by the acceptor protein. HscA binds to a conserved ${ }^{99}$ LPPVK $^{103}$ sequence $(7,8,11)$ positioned close to conserved Cys-106 that may be involved in cluster coordination. Binding of $\mathrm{Isc}_{2}[2 \mathrm{Fe} 2 \mathrm{~S}]$ to $\mathrm{HscA}$ alone does not appear to significantly affect the properties of the cluster or make it more available for transfer, but conformational changes coupled to the HscA $\mathrm{T} \Longrightarrow \mathrm{R}$ transition during ATP hydrolysis may act to make the cluster more available. The $\mathrm{CD}$ changes observed using limiting concentrations of ATP indicate that $\mathrm{Isc}^{*}$ formation requires only a single turnover, and hence Isc $\mathrm{U}^{*}$ will be formed during each HscA ATPase cycle. The CD results obtained using limiting concentrations of $\mathrm{HscA}$ further indicate that only one HscA is required to affect $\mathrm{Isc}_{2}[2 \mathrm{Fe} 2 \mathrm{~S}]$, and the scheme therefore shows only one IscU molecule of the dimer as Isc U*. It is possible, however, that effects on one IscU monomer could have effects on the other monomer of the dimer complex as well.

Whether the cochaperone $\mathrm{HscB}$ plays a direct role in cluster activation is not clear from the present studies. $\mathrm{HscB}$ enhances the binding of IscU to HscA and synergistically stimulates the rate of HscA ATP hydrolysis, but the extent of the interactions between $\mathrm{HscB}$ and $\mathrm{IscU}$ in the ternary $\mathrm{HscA}(\mathrm{ATP})-\mathrm{HscB}-\mathrm{Isc}_{2}[2 \mathrm{Fe} 2 \mathrm{~S}]$ complex and whether these contribute to any changes in IscU structure during the HscA T $\Rightarrow$ R transition associated with ATP hydrolysis have not been characterized. Recent studies of $\mathrm{HscB}-\mathrm{IscU}$ interactions have revealed that mutations in the IscU binding region of $\mathrm{HscB}$ decrease allosteric cooperativity within the $\mathrm{HscA}-\mathrm{HscB}-\mathrm{IscU}$ complex (29). This finding suggests that $\mathrm{HscB}-\mathrm{IscU}$ interactions are coupled to the $\mathrm{T} \Rightarrow \mathrm{R}$ conformational changes of $\mathrm{HscA}$ and these interactions could play an essential role in $\mathrm{IscU}^{*}$ formation.

\footnotetext{
${ }^{3}$ Because the rate of cluster relaxation, i.e., IscU* $\Longrightarrow \mathrm{IscU}$, is significantly slower than ATP hydrolysis, it is possible that Isc $U^{*}$ may rebind to $\mathrm{HscA}$, but the affinities of $\mathrm{HscA}$ and $\mathrm{HscB}$ for $\mathrm{IscU}^{*}$ are not known.
}

The finding that $\mathrm{HscA}$ and $\mathrm{HscB}$ accelerate $\mathrm{Isc}_{2}[2 \mathrm{Fe} 2 \mathrm{~S}]$ cluster transfer raises the question of whether transfer occurs while the $\mathrm{IscU}^{*}$ is bound to $\mathrm{HscA}$ or subsequent to its release. Some insights into this question can be gleaned by analysis of the kinetics of the HscA ATPase cycle, the rate of activated cluster relaxation, and the rate of cluster transfer. The rate-determining step of the HscA ATPase cycle is the hydrolysis of ATP, and $k_{\text {cat }}$ for this step is $\sim 1 \mathrm{~s}^{-1}$ (17). The rate of IscU activation, i.e., IscU $\Longrightarrow$ Isc ${ }^{*}$ (Figure 7), is presumed to occur at a similar rate consistent with the rapid initial CD changes observed in Figures 5 and 6. ATP hydrolysis, nucleotide exchange, and conversion of HscA to the low-affinity T-state state occur rapidly, and this favors the release of Isc $U^{*}$. Under typical physiological conditions HscA will exist predominantly as the HscA(ATP) T-state complex or as the $\mathrm{HscA}(\mathrm{ATP})-\mathrm{HscB}-\mathrm{Isc}_{2}[2 \mathrm{Fe} 2 \mathrm{~S}]$ complex. ${ }^{3}$ For this reason it seems most likely that cluster transfer will occur from free $\mathrm{IscU}^{*}$ rather than from Isc $\mathrm{U}^{*}$ bound to the chaperone. The rate of cluster relaxation observed in the CD changes of Figures 5 and $6, \sim 0.1 \mathrm{~s}^{-1}$, is also faster than the rate of cluster transfer, and this suggests that multiple ATPase cycles to form IscU* are likely to occur prior to transfer.

\section{SUPPORTING INFORMATION AVAILABLE}

Figures showing (1) the binding of IscU to HscA(T212V) monitored by isothermal titration calorimetry and (2) the effect of ATP on the absorption spectrum of HscA(T212V). This material is available free of charge via the Internet at http://pubs.acs.org.

\section{REFERENCES}

1. Johnson, D. C., Dean, D. R., Smith, A. D., and Johnson, M. K. (2005) Structure, function, and formation of biological iron-sulfur clusters. Annu. Rev. Biochem. 74, 247-281.

2. Rouault, T. A., and Tong, W. H. (2005) Iron-sulphur cluster biogenesis and mitochondrial iron homeostasis. Nat. Rev. Mol. Cell Biol. 6, 345-351.

3. Fontecave, M., and Ollagnier-de-Choudens, S. (2008) Iron-sulfur cluster biosynthesis in bacteria: mechanisms of cluster assembly and transfer. Arch. Biochem. Biophys. 474, 226-237.

4. Lill, R., and Mühlenhoff, U. (2008) Maturation of iron-sulfur proteins in eukaryotes: Mechanisms, connected processes, and diseases. Annu. Rev. Biochem. 77, 669-700.

5. Ayala-Castro, C., Saini, A., and Outten, F. W. (2008) Fe-S cluster assembly pathways in bacteria. Microbiol. Mol. Biol. Rev. 72, 1101125.

6. Vickery, L. E., and Cupp-Vickery, J. R. (2007) Molecular chaperones $\mathrm{HscA} / \mathrm{Ssq} 1$ and $\mathrm{HscB} / \mathrm{Jac} 1$ and their roles in iron-sulfur protein maturation. Crit. Rev. Biochem. Mol. Biol. 42, 95-111.

7. Hoff, K. G., Ta, D. T., Tapley, T. L., Silberg, J. J., and Vickery, L. E. (2002) Hsc66 substrate specificity is directed toward a discrete region of the iron-sulfur cluster template protein IscU. J. Biol. Chem. 277, 27353-27359.

8. Hoff, K. G., Cupp-Vickery, J. R., and Vickery, L. E. (2003) Contributions of the LPPVK motif of the iron-sulfur template protein IscU to interactions with the Hsc66-Hsc20 chaperone system. J. Biol. Chem. 278, 37582-37589.

9. Dutkiewicz, R., Schilke, B., Knieszner, H., Walter, W., Craig, E. A., and Marszalek, J. (2003) Ssq1, a mitochondrial Hsp70 involved in iron-sulfur $(\mathrm{Fe} / \mathrm{S})$ center biogenesis. Similarities to and differences from its bacterial counterpart. J. Biol. Chem. 278, 2971929727.

10. Dutkiewicz, R., Schilke, B., Cheng, S., Knieszner, H., Craig, E. A., and Marszalek, J. (2004) Sequence-specific interaction between mitochondrial Fe-S scaffold protein Isu and Hsp70 Ssq1 is essential for their in vivo function. J. Biol. Chem. 279, 29167-29174.

11. Cupp-Vickery, J. R., Peterson, J. C., Ta, D. T., and Vickery, L. E. (2004). Crystal structure of the molecular chaperone HscA substrate 
binding domain complexed with the IscU recognition peptide ELPPVKIHC. J. Mol. Biol. 342, 265-1278.

12. Hoff, K. G., Silberg, J. J., and Vickery, L. E. (2000) Interaction of the iron-sulfur cluster assembly protein IscU with the Hsc66/Hsc20 molecular chaperone system of Escherichia coli. Proc. Natl. Acad. Sci. U.S.A. 97, 7790-7795.

13. Mühlenhoff, U., Gerber, J., Richhardt, N., and Lill, R. (2003) Components involved in assembly and dislocation of iron-sulfur clusters on the scaffold protein Isu1p. EMBO J. 22, 4815-4825.

14. Dutkiewicz, R., Marszalek, J., Schilke, B., Craig, E. A., Lill, R., and Muhlenhoff, U. (2006) The Hsp70 chaperone Ssq1p is dispensable for iron-sulfur cluster formation on the scaffold protein Isu1p. J. Biol. Chem. 281, 7801-7808.

15. Bonomi, F., Iametti, S., Ta, D. T. and Vickery, L. E. (2005) The E. coli $\mathrm{HscA} / \mathrm{HscB}$ chaperone/cochaperone system facilitates iron-sulfur cluster transfer from holo-IscU to apo-ferredoxin in an ATP-dependent manner, http://www.umich.edu/ icbic/ Abstracts/370469-1.pdf.

16. Chandramouli, K., and Johnson, M. K. (2006) HscA and HscB stimulate [2Fe-2S] cluster transfer from IscU to apoferredoxin in an ATP-dependent reaction. Biochemistry 45, 11087-11095.

17. Silberg, J. J., Tapley, T. L., Hoff, K. G., and Vickery, L. E. (2004) Regulation of the HscA ATPase reaction cycle by the co-chaperone $\mathrm{HscB}$ and the iron-sulfur cluster assembly protein IscU. J. Biol. Chem. 279, 53924-53931.

18. Ta, D. T., and Vickery, L. E. (1992) Cloning, sequencing, and overexpression of a $[2 \mathrm{Fe}-2 \mathrm{~S}]$ ferredoxin gene from. Escherichia coli. J. Biol. Chem. 267, 11120-11125.

19. Vickery, L. E., Silberg, J. J., and Ta, D. T. (1997) Hsc66 and Hsc20, a new heat shock cognate molecular chaperone system from Escherichia coli. Protein Sci. 6, 1047-1056.
20. Agar, J. N., Krebs, C., Frazzon, J., Huynh, B. H., Dean, D. R., and Johnson, M. K. (2000) IscU as a scaffold for iron-sulfur cluster biosynthesis: sequential assembly of [2Fe-2S] and [4Fe-4S] clusters in IscU. Biochemistry 39, 7856-7862.

21. Bonomi, F., Iametti, S., Ta, D. T., and Vickery, L. E. (2005) Multiple turnover transfer of [2Fe2S] clusters by the iron-sulfur cluster assembly scaffold proteins IscU and IscA. J. Biol. Chem. 280, 29513-29518.

22. McCarty, J. S., and Walker, G. C. (1991) DnaK as a thermometer: Threonine-199 is site of autophosphorylation and is critical for ATPase activity. Proc. Natl. Acad. Sci. U.S.A. 88, 9513-9517.

23. Barthel, T. T., and Walker, G. C. (2001) ATPase-defective derivatives of Escherichia coli DnaK that behave differently with respect to ATP-induced conformational change and peptide release. J. Bacteriol. 183, 5482-5490.

24. Silberg, J. J., and Vickery, L. E. (2000) Kinetic characterization of the ATPase cycle of the molecular chaperone Hsc66 from Escherichia coli. J. Biol. Chem. 275, 7779-7786.

25. Silberg, J. J., Hoff, K. G., Tapley, T. L., and Vickery, L. E. (2001) The $\mathrm{Fe} / \mathrm{S}$ assembly protein IscU behaves as a substrate for the molecular chaperone Hsc66 from Escherichia coli. J. Biol. Chem. 276, 1696-1700.

26. Wu, G., Mansy, S. S., Wu, S. P., Sureus, K. K., Foster, M. W., and Cowan, J. A. (2002) Characterization of an iron-sulfur cluster assembly protein (ISU1) from Schizosaccharomyces pombe. Biochemistry 41, 5024-5032.

27. Füzéry, A. K., Tonelli, M., Ta, D. T., Cornilescu, G., Vickery, L. E., and Markley, J. L. (2008) Solution structure of the ironsulfur cluster cochaperone $\mathrm{HscB}$ and its binding surface for the iron-sulfur assembly scaffold protein IscU. Biochemistry 47, 9394-9404.

BI801565J 